\title{
Measurement of isolated photons with and without accompanying jets at HERA
}

\author{
Andrii ludin ${ }^{1}$ \\ (for the ZEUS Collaboration) \\ Deutsches Elektronen-Synchrotron, Notkestraße 85, 22607 Hamburg, Germany \\ National Technical University of Ukraine "Kyiv Polytechnic Institute" \\ E-mail: andriy.iudinedesy.de
}

Isolated-photon+jet production in Deep Inelastic Scattering ep collisions at a centre-of-mass energy of $318 \mathrm{GeV}$ has been measured using the ZEUS detector at HERA with an integrated luminosity of $326 \mathrm{pb}^{-1}[1,2]$. Measured cross sections are presented as functions of the photon and the jet transverse energy and pseudorapidity in isolated-photon transverse-energy and pseudorapidity ranges $4<E_{T}^{\gamma}<15 \mathrm{GeV}$ and $-0.7<\eta^{\gamma}<0.9$, jet transverse-energy and pseudorapidity ranges $2.5<E_{T}^{\text {jet }}<35 \mathrm{GeV}$ and $-1.5<\eta^{\text {jet }}<1.8$, for exchanged-photon virtualities $10<Q^{2}<350 \mathrm{GeV}^{2}$. Parton-shower Monte Carlo predictions and perturbative QCD calculations are compared to the data. Isolated-photon production in photoproduction, both inclusive and together with a jet, has also been measured using an integrated luminosity of 370 $\mathrm{pb}^{-1}$. Measurements were made in ranges $6<E_{T}^{\gamma}<15 \mathrm{GeV},-0.7<\eta^{\gamma}<0.9,4<E_{T}^{\text {jet }}<35 \mathrm{GeV}$, $1.5<\eta^{\text {jet }}<1.8$ and $Q^{2}<1 \mathrm{GeV}^{2}$. Differential cross sections are presented for inclusive isolated photon production as functions of the transverse momentum and pseudorapidity of the photon and the jet. Higher-order theoretical calculations are compared to the results [1].

XXI International Workshop on Deep-Inelastic Scattering and Related Subjects April 22-26, 2013

Marseilles, France

1

Speaker 


\section{Introduction}

Two kinematic regions can be distinguished at an electron-proton collider such as HERA: photoproduction (PHP), where the exchanged photon is quasi-real with almost on-mass-shell virtuality $Q^{2} \leq 1 \mathrm{GeV}^{2}$; deep inelastic scattering (DIS), where the electron interacts with a parton from the proton via the exchange of a virtual boson with large virtuality $Q^{2} \geq 1 \mathrm{GeV}^{2}$.

A prompt photon is one that emerges directly from a perturbative quantum chromodynamics (pQCD) process. Prompt photons are a useful tool to test QCD models and can be used to measure and constrain the parton densities of the proton and photon.

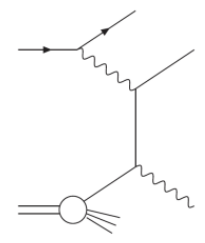

a)

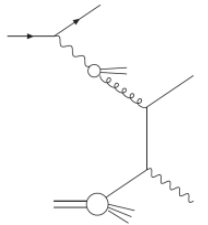

b)

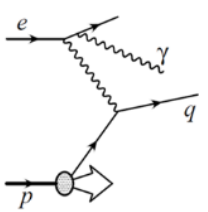

c)

Figure 1: "Direct" (a) and "resolved" photoproduction processes (b) and "LL" (c) mechanisms of isolated photon production.

Fig. 1 gives examples of the lowest-order (LO) diagrams for high-energy photon photoproduction in quantum chromodynamics (QCD). The "direct" production process (a) has the entire incoming photon absorbed by a quark from the incoming proton, while in "resolved" processes (b), the photon's hadronic structure provides a quark or gluon that interacts with a parton from the proton. In DIS, photons are emitted directly by quarks ("QQ" photons) or leptons ("LL" photons, Fig. 1 (c)) that take part in a hard scattering process.

In this report, recent measurements of isolated photons with and without accompanying jet requirement in PHP and isolated photons with jet requirement in DIS at HERA performed with the ZEUS detector are presented. The measurements presented here correspond to integrated luminosities of $370 \mathrm{pb}^{-1}$ for the PHP and of $326 \mathrm{pb}^{-1}$ for the DIS analysis, respectively.

\section{Isolated photons with and without jet requirement in photoproduction}

\subsection{Event Selection and extraction of the signal}

The event selection was such that $Q^{2}<1 \mathrm{GeV}^{2}$ and no scattered beam electron was permitted. Energy-flow objects (EFOs) [3] were constructed from clusters of calorimeter (CAL) cells and tracks. Photon candidates were identified as trackless EFOs for which at least $90 \%$ of the reconstructed energy was measured in the electromagnetic CAL cell.

The reconstructed transverse energy of the photon candidate, $E_{T}^{\gamma}$, was required to lie within the range $6<E_{T}^{\gamma}<15 \mathrm{GeV}$ and the pseudorapidity, $\eta^{\gamma}$, had to be $-0.7<\eta^{\gamma}<0.9$. To reduce the background from neutral mesons and converted electrons, isolation criteria were applied. To reduce the fraction of fragmentation events, it was required that the ratio of the photon energy to the energy of the jet-like structure containing the photon was greater than 0.9. 
Jet reconstruction was performed with all EFOs in the event using the $k_{T}$ clustering algorithm [4] in the $E$-scheme in the longitudinally invariant inclusive mode [5], with the $R$ parameter set to 1.0. The jets were required to be such that $4<E_{T}^{\text {jet }}<35 \mathrm{GeV}$ and $-1.5<\eta^{\text {jet }}<$ 1.8. One of the jets found by this procedure corresponds to or includes the photon candidate. An additional accompanying jet was required; if more than one was found, that with the highest $E_{T}^{\text {jet }}$ was used.

ZEUS

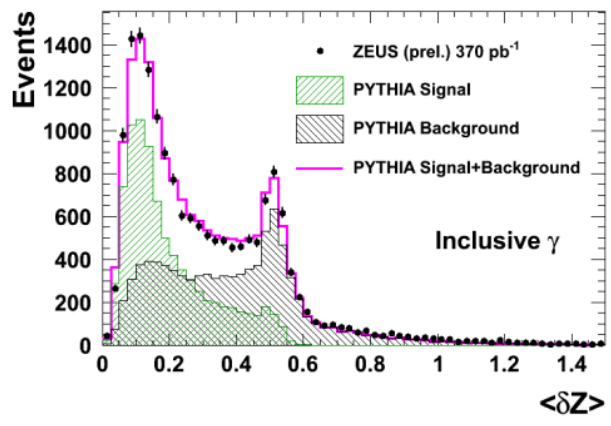

a)
ZEUS

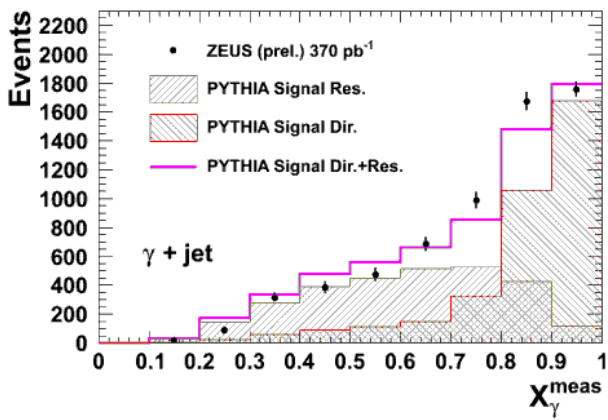

b)

Figure 2: Distribution of (a) $<\delta Z>$ and (b) events detected for different values of $x_{\gamma}{ }^{\text {meas }}$, compared to a mixture of Pythia-generated direct and resolved events (b).

For the signal extraction of isolated photons a shower shape technique was used. The variable $\langle\delta Z\rangle=\Sigma_{i}\left|Z_{i}-Z_{\text {cluster }}\right| /\left(\mathrm{w}_{\text {cell }} \Sigma_{i} E_{i}\right)$ was used to describe the shower shape; its distribution is shown in Fig. 2 (a). Here $Z_{\mathrm{i}}$ is the $Z$ position of the centre of the $i$-th cell, $Z_{\text {cluster }}$ is the $Z$ position of the centre of the CAL cluster where the photon candidate deposited its energy, $\mathrm{w}_{\text {cell }}$ is the cell width in the $Z$ direction, and $E_{i}$ is the energy deposited in the cell. The sum runs over the CAL cells of the cluster. The $<\delta Z>$ distribution shows a two-peak structure with first peak at $\approx 0.1$ corresponding to the signal and second at $\approx 0.5$ to the background.

To evaluate the acceptances, a ratio between direct and resolved components as modelled by Pythia is evaluated. These components can be distinguished by examination of the quantity $x_{\gamma}^{\text {meas }}=\left(E^{\gamma}+E^{\text {jet }}-p_{Z}^{\gamma}-p_{Z}^{\text {jet }}\right) /\left(E^{\text {all }}-p_{Z}^{\text {all }}\right)-$ a measure of the fraction of the incoming photon energy given to the final state photon and jet. Fig. 2 (b) shows the numbers of events in different bins of $x_{\gamma}{ }^{\text {meas }}$; a peak close to unity can be attributed to direct events, and a tail at lower values due to resolved events.

\subsection{Theory}

Two theoretical predictions are compared to the measurements. The default renormalisation scale and factorisation scales are taken in the isolated-photon transverse-energy $\mu_{R}=\mu_{F}=E_{T}^{\gamma}$. In the approach of FGH [6], the LO and NLO diagrams and the box diagram term are calculated explicitly. Fragmentation processes are calculated in terms of a fragmentation function in which a quark or gluon gives rise to a photon. Theoretical uncertainties arise due to the choice of the renormalisation scale, and were estimated by varying this scale by factors of 0.5 and 2.0. The $k_{T}$ factorisation method used by LZ [7] is at LO but makes use of unintegrated parton densities in the proton, and gives a quark-radiated contribution that is enhanced relative 
to the leading-order collinear approximations. Uncertainties come from renormalisation and factorisation scales varied by factors 0.5 and 2 simultaneously.

ZEUS

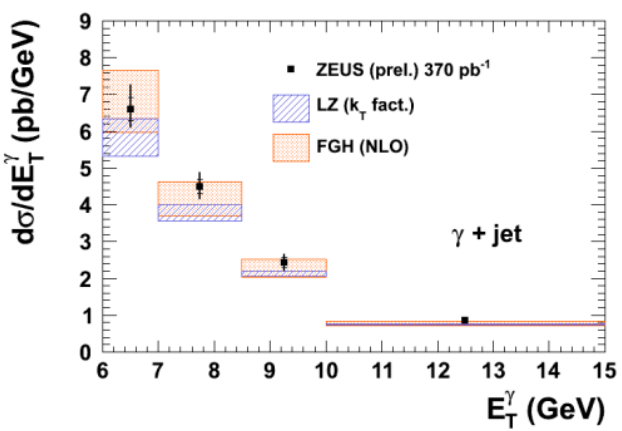

a)

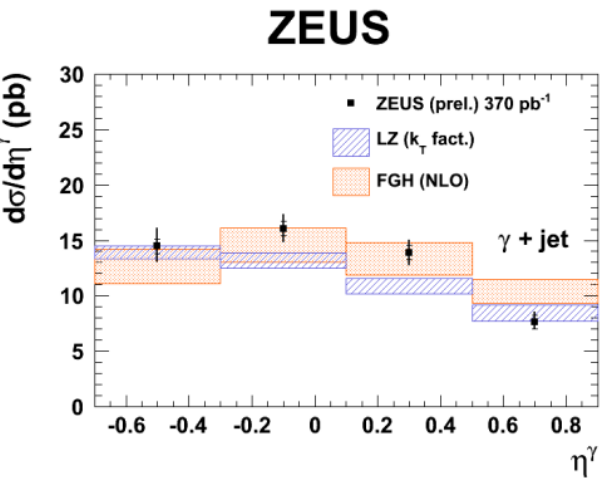

b)

Figure 3: Cross sections as a function of (a) $E_{T}^{\gamma}$ and (b) $\eta^{\gamma}$ for inclusive photon in PHP compared to predictions from FGH and LZ.

\subsection{Results}

Differential cross sections for the production of an isolated photon, with and without requirement of at least one additional jet were measured within the kinematic region defined by $Q^{2}<1 \mathrm{GeV}^{2},-0.7<\eta^{\gamma}<0.9,6<E_{T}^{\gamma}<15 \mathrm{GeV}, 4<E_{T}^{\text {jet }}<35 \mathrm{GeV}$ and $-1.5<\eta^{\text {jet }}<1.8$ in the laboratory frame. The differential cross sections as functions of $\mathrm{E}_{\mathrm{T}}^{\gamma}$ and $\eta^{\gamma}$ are shown in Fig. 3. FGH predictions provide good description of all the distributions within the uncertainties. A reasonable description is also provided by the $k_{T}$ factorisation model of LZ.

\section{Isolated photons with and without jet requirement in DIS}

\subsection{Event Selection and extraction of the signal}

Events were selected by requiring a scattered-electron candidate with a polar angle $\theta_{e}>$ $140^{\circ}$ and energy $E_{e}>10 \mathrm{GeV}$. The kinematic region $10<Q^{2}<350 \mathrm{GeV}^{2}$ was selected. The photon candidate, which is measured as a cluster of cells with signals in the CAL, was required to have $4<E_{T}^{\gamma}<15 \mathrm{GeV},-0.7<\eta^{\gamma}<0.9$ and to be responsible for at least $90 \%$ of the energy of the jet-like structure that contained it. Jets were selected with $E_{T}^{\text {jet }}>2.5 \mathrm{GeV}$ and $-1.5<\eta^{\text {jet }}<$ 1.8 and clustered using the $k_{T}$ algorithm.

For the signal extraction of isolated photons a shower shape method based on $<\delta Z>$ quantity was used as described above.

\subsection{Theory}

The results are compared to theoretical calculations by GKS [8] and BLZ [9]. The theoretical uncertainties due to the choice of the renormalisation and factorisation scales $\mu_{F}=\mu_{R}$ $=\left(Q^{2}+\left(p_{T}^{\text {jet }}\right)^{2}\right)^{1 / 2}$ were evaluated by scaling $\mu_{R}$ and $\mu_{F}$ by a factor 2 up and down. GKS predictions are fixed order NLO calculations. They take into account both LL and QQ contributions as well as the LQ interference term. The BLZ predictions are made in the framework of the QCD $k_{T}$ factorisation approach with unintegrated proton parton densities. 


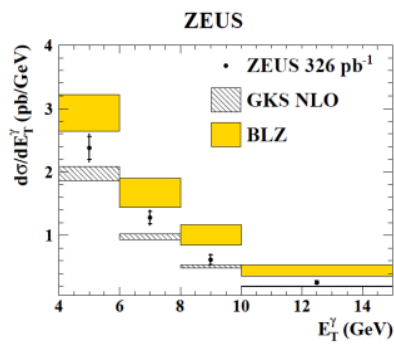

a)

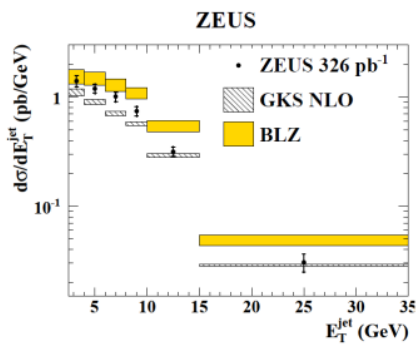

d)

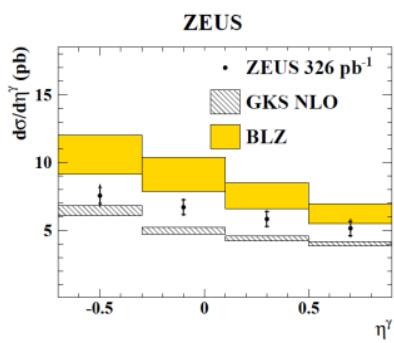

b)

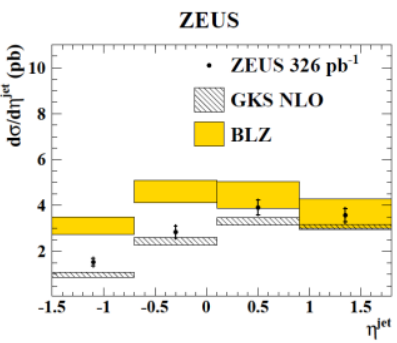

e)

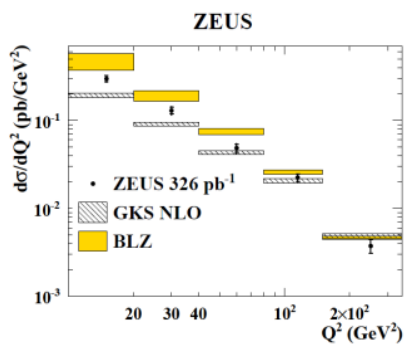

c)

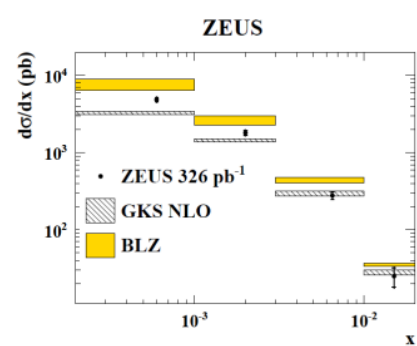

f)

Figure 4: Cross sections as a function of (a) $E_{T}^{\gamma}$, (b) $\eta^{\gamma}$, (c) $Q^{2}$, (d) $E_{T}^{\text {jet }}$, (e) $\eta^{\text {jet }}$ and (f) $x$ for isolated photon accompanyied by hadronic jet in DIS compared to predictions from GKS and BLZ.

\subsection{Results}

In Fig. 4 differential cross sections as function of $E_{T}^{\gamma}, \eta^{\gamma}, Q^{2}, E_{T}^{\text {jet }}, \eta^{\text {jet }}$ and $x$ are presented in the kinematic region defined by $E_{e}>10 \mathrm{GeV}, 10<Q^{2}<350 \mathrm{GeV}^{2}, 4<E_{T}^{\gamma}<15 \mathrm{GeV},-0.7<$ $\eta^{\gamma}<0.9, E_{T}^{\text {jet }}>2.5 \mathrm{GeV}$ and $-1.5<\eta^{\text {jet }}<1.8$. Both theories provide a reasonable description of the data in shape. The GKS predictions systematically underestimate the data by typically $20 \%$ while the BLZ predictions overestimate them by about $20 \%$. The results indicate the need for further improved QCD calculations.

\section{References}

[1] Proc. Photon 2013, LPNHE, Paris, 2013, Proc. of Sci. (to be published)

[2] ZEUS Collaboration, H. Abramowicz et al., Phys. Lett. B 715 (2012) 88

[3] ZEUS Collaboration, J. Breitweg et al., Eur. Phys. J. C 1 (1998) 81

[4] S. Catani et al., Nucl. Phys. B 406 (1993) 187.

[5] S.D. Ellis and D.E. Soper, Phys. Rev. D 48 (1993) 3160

[6] M. Fontannaz, J.Ph. Guillet and G. Heinrich, Eur. Phys. J. C 21 (2001) 303)

[7] A. Lipatov and N. Zotov, Phys. Rev. D 81 (2010) 094027

[8] A. Gehrmann-De Ridder, G. Kramer and H. Spiesberger, Nucl. Phys. B 578 (2000) 326

[9] S. Baranov, A. Lipatov and N. Zotov, Phys. Rev. D 81 (2010) 094034 